\title{
Zur Hemmung der aktiven lonenaufnahme durdh Arsenat
}

\author{
Von Josef WeIgL \\ Aus dem Botanischen Institut der Technischen Hochschule Darmstadt \\ IZ. Naturforschg. 19 b, 646-648 [1964] ; eingegangen am 17. Februar 1964)
}

\begin{abstract}
Durch Vergiftung mit Arsenat in steigender Konzentration wird die Atmung von Maiswurzeln zunehmend erhöht und ihr Gehalt an labilem Nucleotidphosphat und organischen Phosphatverbindungen in zunehmendem Maße herabgedrückt. Gleichlaufend damit wird ihre Fähigkeit zur aktiven Ionenaufnahme gelähmt. Die Befunde werden als Beweis für die Wirkung von „energiereichen“ Phosphatverbindungen (letztlich von ATP!) beim Vollzug der aktiven (endergonischen) Ionenaufnahme angesehen.
\end{abstract}

Die lähmende Wirkung von Arsenat auf die aktive Ionenaufnahme durch pflanzliches Gewebe wurde von $\mathrm{Arisz}^{1}$ für $\mathrm{Cl}^{\ominus}$, von $\mathrm{Higginbotham}^{2}$ und MENGEL ${ }^{3}$ für $\mathrm{Rb}^{\oplus}$ nachgewiesen. $\mathrm{W}_{\mathrm{EIGL}}{ }^{4}$ fand, daß die Hemmung der $\mathrm{PO}_{4}{ }^{3 \ominus}$. und $\mathrm{SO}_{4}{ }^{2 \ominus}$-Aufnahme der Unterdrückung der oxydativen Phosphorylierung (gemessen als Einbau von ${ }^{32} \mathrm{P}$ in ATP durch Maiswurzeln nach Vergiftung mit verschiedenen Arsenatkonzentrationen) parallel geht. Da die Atmung bei den verwendeten Arsenatkonzentrationen nicht negativ beeinflußt, sondern gesteigert wird ${ }^{2,4}$, kann man den Schluß ziehen, daß die aktive $\mathrm{Rb}^{\oplus}$ - und $\mathrm{SO}_{4}{ }^{2}{ }^{2}$. Aufnahme nicht mit den Redoxschritten der Atmungskette verknüpft ist ${ }^{3}$, sondern durch das Ausmaß der oxydativen Phosphorylierung begrenzt wird. (Bei der Hemmung der aktiven Phosphataufnahme ist sicherlich eine kompetitive Wirkung des Arsenats an den Transportorten der Membranen nicht auszuschlieBen.) In der vorliegenden Mitteilung wird eine Korrelation der aktiven $\mathrm{Cl}^{\ominus}$ - und $\mathrm{SO}_{4}{ }^{2 \ominus}$-Aufnahme zum Gehalt an „energiereichem" Phosphat $(\sim \mathrm{P})$ und an (unter Aufwand von $\sim \mathrm{P}$ gebildeten) Phosphatverbindungen in arsenatvergifteten Wurzeln beschrieben.

\section{Versuche}

Maissamen wurden angekeimt und wie früher ${ }^{4}$ in der ${ }^{31}$ P-freien Nährlösung bis zu einer Wurzellänge von $15-20 \mathrm{~cm}$ kultiviert. Ein Teil der Wurzeln wurde in Nährlösung gezogen, die $0,1 \mathrm{mC}$ trägerfreies ${ }^{32} \mathrm{PO}_{4}{ }^{3}{ }^{\ominus} / 100 \mathrm{ml}$ enthielt. Nach der Kultur wurden die Wurzeln in inaktiver Nährlösung gewaschen, bis die Waschflüssigkeit frei von Radioaktivität war und einen Tag in der inaktiven Nährlösung weiterkultiviert. Je $650,8 \mathrm{~cm}$ lange Segmente der markierten und der nicht markierten Wurzeln wurden erneut gewaschen und 8 Stdn. mit $10 \mathrm{ml} 0,10^{-4}, 10^{-3}$ und $10^{-2}-\mathrm{m}$.

1 W. H. Arisz, Acta bot. neer. 7, 1 [1958].

2 N. Higginbotham, Plant Physiol. 34, 645 [1959].
$\mathrm{Na}_{2} \mathrm{HAsO}_{4}$-Lösung inkubiert. Am Ende der Vergiftung waren (durch Austauschdiffusion?) folgende Phosphatmengen aus den ${ }^{32} \mathrm{P}$-markierten Segmenten in die Nährlösung ausgetreten: $2,4 \cdot 10^{3} \mathrm{Imp} / \mathrm{min}$ in die arsenatfreie Nährlösung, $5 \cdot 10^{3} \mathrm{Imp} / \mathrm{min}$ bei $10^{-4}-\mathrm{m} ., 10 \cdot 10^{3}$ $\mathrm{Imp} / \mathrm{min}$ bei $10^{-3}-\mathrm{m}$. und $21 \cdot 10^{3} \mathrm{Imp} / \mathrm{min}$ bei $10^{-2}-\mathrm{m}$. Arsenat. Die Segmente wurden 20 min gewaschen und dann die folgenden Experimente damit ausgeführt:

\section{Mit ${ }^{32}$ P-freien Segmenten}

a) Manometrische Messung der Atmung in $10^{-5}-m$. $\mathrm{K}_{2} \mathrm{SO}_{4}$ während der folgenden 2 Stunden.

b) Messung der aktiven $\mathrm{SO}_{4}{ }^{2 \ominus}$-Aufnahme aus einer $10^{-5}$-m. Lösung von $\mathrm{K}_{2} \mathrm{SO}_{4}\left(+0,001 \mathrm{mC}{ }^{35} \mathrm{SO}_{4}{ }^{2 \ominus} / \mathrm{ml}\right)$ während 2 Stunden. Die Segmente wurden nach der Aufnahmephase $10 \mathrm{~min}$ gewaschen, homogenisiert und ihre Radioaktivität ermittelt.

c) Messung der aktiven $\mathrm{Cl}^{\ominus}$-Aufnahme aus einer $10^{-5}$ - $m$. Lösung von $\mathrm{KCl}\left(2,8 \cdot 10^{4} \mathrm{Imp} / \mathrm{min} / \mathrm{ml}\right)$ während 2 Stunden. Das Waschen der Segmente und die Ermittlung ihrer Radioaktivität erfolgte wie bei Versuch 1 b. Die Ergebnisse sind in Abb. 1 wiedergegeben.

\section{Mit ${ }^{32}$ P-markierten Segmenten}

Je 65 Segmente wurden in Methanol homogenisiert und das Homogenat mit einem kalten Luftstrom eingetrocknet. Der Rückstand wurde in $4 \mathrm{ml}$ Wasser aufgenommen und zentrifugiert. Mit je $0,5 \mathrm{ml}$ des Überstandes wurden folgende Gesamtradioaktivitäten ermittelt: $22,9 \cdot 10^{4} \mathrm{Imp} / \mathrm{min}$ bei unvergifteten Wurzeln, $22,8 \cdot 10^{4}$ $\mathrm{Imp} / \mathrm{min}$ bei $10^{-4}-\mathrm{m} ., 21,3 \cdot 10^{4} \mathrm{Imp} / \mathrm{min}$ bei $10^{-3}-\mathrm{m}$. und $21,7 \cdot 10^{4} \mathrm{Imp} / \mathrm{min}$ bei $10^{-2}-\mathrm{m}$. Arsenat. Je $1 \mathrm{ml}$ des Überstehenden wurde gefriergetrocknet und in n-Butanol-Methanol-Eisessig-Wasser 156:75:40:104 22 Stdn. aufsteigend chromatographiert ${ }^{4}$; von dem Extrakt aus unvergifteten Wurzeln wurden nur $0.2 \mathrm{ml}$ zur Chromatographie verwandt. Die Auswertung der Chromatogramme erfolgte mit dem Radiopapierchromatographen $(\mathrm{F} \& \mathrm{H})$ und durch Planimetrieren der Gipfel (Abb. 1 und 2). Je 1,5 $\mathrm{ml}$ des Überstehenden wurde zweidimensional in n-Butanol-Methanol-Eisessig-Wasser 156:75:40:104 (22 Stdn.) und Methanol-m. Ammoniumacetat $7: 3$ (9 Stdn.) aufsteigend chromatographiert und das die Nucleotide enthaltende (bei $\mathrm{W}_{\text {EIGL }}{ }^{4}$

\footnotetext{
3 K. Mengel, Physiol. Plantarium [Copenhagen] 16, 767 [1963].

4 J. WeIGL, Planta 60, 307 [1963].
} 


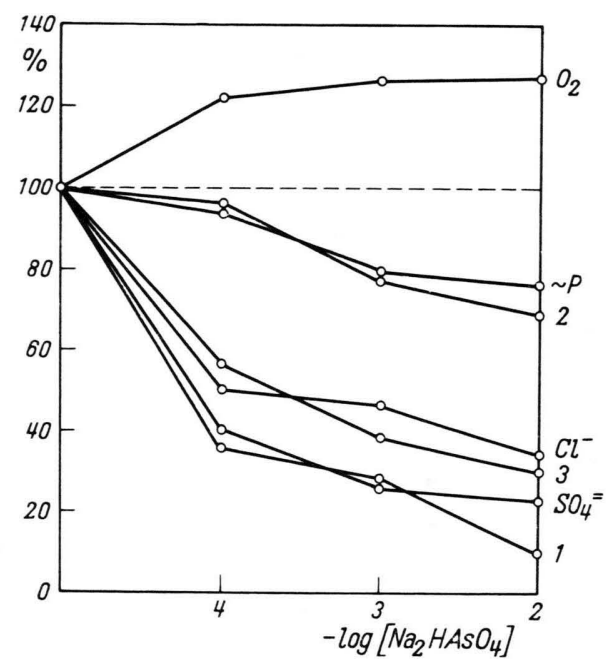

Abb. 1. Der Einfluß von Arsenat auf die aktive Aufnahme von Sulfat und Chlorid, auf die $\mathrm{O}_{2}$-Aufnahme und auf den Gehalt an $\sim \mathrm{P}$ und einigen Phosphatfraktionen. Die Wurzelsegmente wurden $8 \mathrm{Stdn}$. in Arsenatlösungen der angegebenen Konzentration vorvergiftet, dann 20 min gewaschen und anschließend die in $2 \mathrm{Stdn}$. erfolgende $\mathrm{Cl}^{\ominus}$. $\mathrm{SO}_{4}{ }^{2 \ominus}$. und $\mathrm{O}_{2}$-Aufnahme gemessen oder extrahiert. Der Extrakt wurde chromatographisch getrennt (vgl. Abb. 2) und der Gehalt an labilem Nucleotidphosphat (überwiegend von ATP, UTP und ADP) ermittelt.

durch eine gestrichelte Linie gekennzeichnete) Papierfeld ohne den Startfleck eluiert. Die Nucleotide wurden an Kohle adsorbiert, nach Crane und Lipmans ${ }^{5}$ hydrolysiert und im Hydrolysat die Radioaktivität ermittelt, die als $\mathrm{Ma}$ für das abgespaltene $\sim \mathrm{P}$ betrachtet wird $(\sim \mathrm{P}$ in Abb. 1$)$.

\section{Besprechung}

Obwohl die Depression des P-Gehaltes der Wurzeln durch Arsenateinwirkung gering ist, so zeigt dennoch die starke Lähmung der unter Verbrauch von $\sim \mathrm{P}$ vollzogenen Synthesen von Phosphatverbindungen (Fraktion 1 und 3, vgl. Abb. 2), daß der Umsatz der „energiereichen“ Phosphate durch Arsenat erheblich gestört wird. Somit wird auch erklärlich, daß bei kurzzeitigem ${ }^{32} \mathrm{PO}_{4}{ }^{3 \ominus}$-Angebot nach Arsenatvergiftung eine sehr starke Unterdrückung des ${ }^{32} \mathrm{P}$-Einbaus in ATP beobachtet wird ${ }^{4}$, wenngleich eingeräumt werden muß, daß diese Hemmung eventuell nicht ausschließlich eine zentrale, von Transportvorgängen unabhängige Störung der oxydativen Phosphorylierung widerspiegelt (deren grundsätzliche Existenz nach den Untersuchungen von GregG und Lehninger ${ }^{6}$ klarer denn je ist). Möglicherweise wird die Verringerung des ${ }^{32} \mathrm{P}$-Einbaus in ATP durch eine kompetitive Hemmung des Antransportes des nach der Vergiftung angebotenen radioaktiven Phosphats verursacht, und zwar durch Arsenat, welches nach der Vergiftungsphase durch Waschen der Wurzeln nicht mehr entfernt werden kann, da es bereits im Plasma oder an der Donnanphase der Plasmaoberfläche gebunden ist und eventuell den Transport des ${ }^{32} \mathrm{PO}_{4}{ }^{3 \ominus}$ durch das Plasmalemma, das Plasma oder die Mitochondrienmembran stört.

Die vorliegenden Versuche zeigen eindeutig, daß bei Arsenateinwirkung die endergonischen Synthesen (z. B. die Synthese der in Fraktion 1 und 3 enthaltenen Phosphatverbindungen) gehemmt sind. Man darf folgern, daß auch die endergonische (unter Aufwand von $\sim \mathrm{P}$ bewerkstelligte) Bildung von zum aktiven Transport fähigen Trägern (bestimmter chemischer Konstitution, räumlicher Gestalt und Anordnung) aus Vorstufen gehemmt ist. Dadurch ergibt

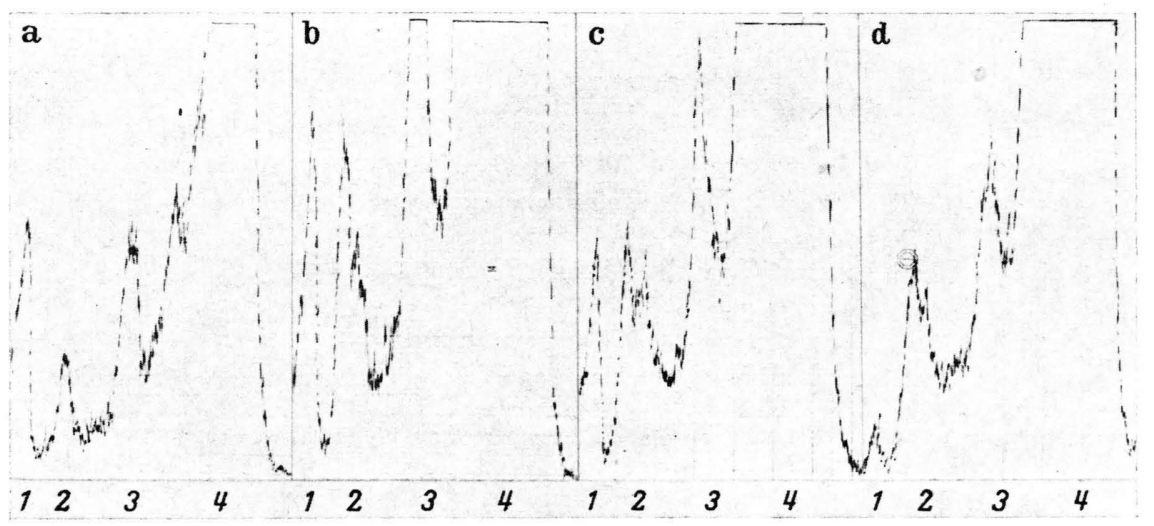

5 R. K. Crane u. F. Lipmann, J. biol. Chemistry 201, 235 [1953].
Abb. 2. Papierchromatographische Trennung der nach Arsenateinwirkung in Maiswurzeln vorliegenden Phophatverbindungen in vier Hauptfraktionen 1, 2, 3 und $4.1=$ unbekannt; $2=$ überwiegend UDPG, ATP, UTP und Diphosphate; 3 = überwiegend Hexosephosphate, (UMP, AMP) ; $4=$ überwiegend anorganisches Phosphat. a) Unvergiftete Wurzeln; 1/20 des Gesamtextraktes. b) $10^{-4}-m$. Arsenat; $1 / 4$ des Gesamtextraktes. c) $10^{-3}-m$. Arsenat; $1 / 4$ des Gesamtextraktes. d) $10^{-2}-m$. Arsenat; $1 / 4$ des Gesamtextraktes.
6 C. T. Gregg u. A. T. Lehninger, Biochim. biophysica Acta [Amsterdam] 78, 27 [1963]. 
sich eine zwanglose Erklärung der Hemmung der aktiven Ionenaufnahme bei ungehemmter Atmung. Die Aufrechterhaltung des Elektronentransportes über die Enzyme der Atmungskette hat zweifellos ihren Grund darin, daß Phosphat durch das verwandte Arsenat wohl schon bei den Primärabläufen der oxydativen Phosphorylierung in kompetitiver Weise vertreten bzw. verdrängt wird. Das in Abb. 3

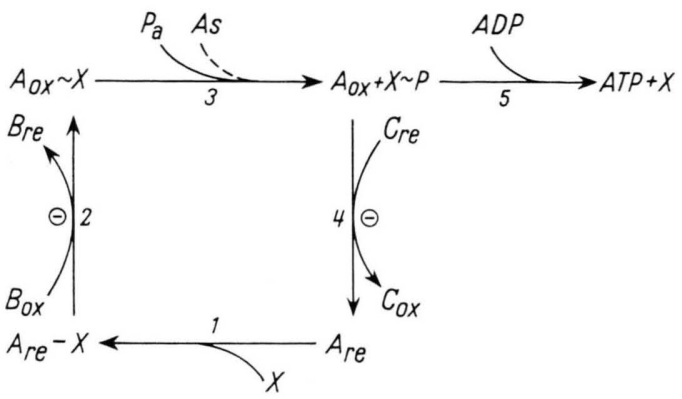

Abb. 3. Schematische Darstellung der Wirkung von Arsenat auf die oxydative Phosphorylierung. Arsenat (As) wird statt anorganischem Phosphat $\left(\mathrm{P}_{\mathrm{a}}\right)$ in die PhosphorylierungsReaktion einbezogen. (Eine „energiereiche“ Arsenatverbindung ist vermutlich nicht existenzfähig.) Arsenat verursacht somit 1. eine Beschleunigung des Elektronenflusses über die Atmungskette (A, B, C) und 2. eine Verminderung der ATP-Bildung, welche letztlich für die Lähmung der aktiven (endergonischen) Ionenaufnahme verantwortlich ist. Nähere Erläuterung im Text.

gegebene Schema wurde in möglichst allgemeiner Form nach Angaben in der Literatur gezeichnet ${ }^{6-8}$, um das Eingreifen von Arsenat (As) bei der oxydativen Phosphorylierung zu veranschaulichen. A, B und $\mathrm{C}$ sind Glieder der Atmungskette im oxydierten (ox) und reduzierten (re) Zustand. $\mathrm{X}$ ist eine unbekannte Intermediärsubstanz, die zusammen mit $A_{\mathrm{re}}$ in einem Oxydoreduktions-Prozeß (Elektronenfluß!) in eine intermediäre „energiereiche“ Bindung $A_{o x} \sim X$ überführt wird, welche ihrerseits im Zuge ihrer exergonischen Spaltung anorganisches Phosphat $\left(\mathrm{P}_{\mathrm{a}}\right)$ in ,energiereiche“ Bindung einführen kann. $A_{o x}$ wird reduziert (Elektronenfluß!) und kehrt zusammen mit $\mathrm{X}$ in den Zyklus zurück. Der Angriffspunkt vieler „Entkoppler“ ist unbekannt. Die Wirkung von DNP auf die oxydative Phosphorylierung wird vielfach in einer Störung der phosphorylierenden Spaltung eines energiereichen Zwischenproduktes (hier $A_{o x} \sim X$ ) gesehen. Anhänger der elektrochemischen Transporttheorien (MEN-

7 C. Cooper u. A. L. Lehninger, J. biol. Chemistry224, 561 [1957].

8 F. Leuthardt, Lehrbuch der phyliologischen Chemie. W. de Gruyter, Berlin 1963.
$\mathrm{GEL}^{3}$, mit weiterer Literatur) nehmen an, daß durch Vermittlung des DNP der Elektronentransport bestimmte Glieder der Atmungskette, deren Oxydoreduktion sowohl für die oxydative Phosphorylierung als auch für die aktive Ionenaufnahme notwendig sein könnte, umgeht. Auch falls sich für den Ablauf der oxydativen Phosphorylierung die Annahme des Intermediärstoffes $\mathrm{X}$ (und somit auch die Bildung von $\mathrm{X} \sim \mathrm{P}$ ) als überflüssig erweisen sollte, falls also $\mathrm{P}_{\mathrm{a}}$ bereits in Reaktion 1 an $\mathrm{A}_{\mathrm{re}}$ angelagert, dann auf die Stufe der energiereichen Bindung gehoben (2) und anschließend unmittelbar auf ADP übertragen wird, muß man dieser Hypothese im Falle der Arsenatwirkung jede Wahrscheinlichkeit absprechen zugunsten der Annahme einer rein kompetitiven Vertretbarkeit von $\mathrm{P}_{\mathrm{a}}$ durch Arsenat. Die vorliegenden Befunde sind somit ein klares Argument für die Rolle von $\sim \mathrm{P}$ als „Energielieferant" bei den endergonen Prozessen der aktiven Ionenaufnahme und gegen die elektrochemischen Theorien der aktiven Ionenaufnahme.

Das Fehlen eines Einflusses von äußerlich zugesetztem ATP auf die aktive Ionenaufnahme durch arsenatvergiftete Wurzeln ${ }^{3}$ konnten wir auch im Falle der $\mathrm{SO}_{4}{ }^{2}{ }^{\ominus}$. und $\mathrm{Cl}^{\ominus}$-Aufnahme feststellen. Die Interpretation dieses Befundes sollte jedoch äußerst vorsichtig betrieben werden, da vor allem sehr unsicher ist, ob äußerlich gebotenes ATP (ungespalten!) an die Orte des Verbrauchs herangeführt werden kann.

Anm. b. d. Korr.: Nach Fertigstellung des Manuskriptes sind Untersuchungen von НемкеR ${ }^{9}$ erschienen, in denen gezeigt wird, daß die entkoppelnde Wirkung der Dinitrophenole auf der Hydrolyse eines „energiereichen“ Zwischenstoffes $\left(A_{o x} \sim X\right)$ beruht, welcher an der Transformation der Energie aus dem Elektronentransport in ATP beteiligt ist. Nach unserer Ansicht muß daraus für die Diskussion der Ionenaufnahme gefolgert werden, daß auch die hemmende Wirkung der Dinitrophenole auf die aktive Ionenaufnahme als eindeutiger Beweis für die (bisher umstrittene ${ }^{3,10}$ ) Annahme gewertet werden darf, daß die aktive Ionenaufnahme direkt oder indirekt durch ATP getrieben wird.

Der Autor dankt Herrn Prof. Dr. H. Ziegler und Herrn Dr. U. Lütrge für die gründliche Erörterung der Arbeit.

\footnotetext{
9 H. C. Hemкer, Biochim. biophysica Acta [Amsterdam] 81 , 1 [1964].

10 R. N. Robertson, Biol. Rev. 35, 231 [1960].
} 